\title{
Graphical Control Tools to Design Control Systems
}

\author{
Huanan Liu ${ }^{1, a,}$, Shujian Zhao, ${ }^{1, b}$ and Dongmin Yu ${ }^{1, c, *}$ \\ ${ }^{1}$ Department of Electrical Engineering, Northeast Electric Power University, Jilin 132012, China \\ a hnliu_neepu@foxmail.com, ${ }^{b} z s j \_n e e p u @$ foxmail.com, ${ }^{c}$ d.yu@neepu.edu.cn, \\ *corresponding author: Dongmin Yu email:d.yu@neepu.edu.cn
}

Keywords: Root Locus; Frequency Tools; Bode Diagram; Nyquist Diagram

\begin{abstract}
Engineers are sometimes asked to analyze the feasibility of existing control systems or design a new system. The conventional analysis and design methods heavily depend on mathematical calculation. In order to minimize calculation, graphical control is proposed. This paper aims to introduce three different graphical control tools (Root Locus, Bode diagram and Nyquist diagram) and then use these tools to analyze and design the control system. Finally, some realistic case studies are used to compare the advantages and disadvantages of graphical tools. The innovation of this paper is adding hand-drawn graphical control to greatly reduce the calculation complexity
\end{abstract}

\section{List of Symbols}

Ts-settling time (s) $\zeta$-damping ratio $\omega_{\mathrm{n}}$-natural frequency $\left(\right.$ rad. $^{-1}$ ) $\%$ OS-percentage of overshoot Ess-steady state error $\mathbf{K}$-gain of the system $\mathbf{Z}_{\mathbf{1}}, \mathbf{Z}_{\mathbf{2}}$-zeros of the system $\mathbf{P}_{\mathbf{1}}, \mathbf{P}_{\mathbf{2}}$-poles of the system $\mathbf{T}_{\mathbf{p}}$-peak time (s) $\mathbf{M}$-magnitude of the phasor $\boldsymbol{\Phi}$-phase angle of phasor (degrees) $\boldsymbol{\Theta}$-angle of asymptote Z-the number of closed loop poles P-the number of open- loop poles $\mathbf{N}$ the number of counter clockwise rotation $\mathbf{P}_{\mathbf{M}^{-}}$-phase margin (degrees) $\mathbf{G}_{\mathbf{M}^{-}}$-gain margin $\mathbf{h}_{\mathbf{1}}, \mathbf{h}_{\mathbf{2}^{-}}$ feedback coefficient

\section{Introduction}

Classical control theory, modern control theory and robust control theory are commonly used today. In advanced engineering and science, automatic control plays a very important role. There are many applications using control systems: examples include rocket firing systems, splashing cooling water systems and car engine systems. Control theory is becoming incredibly important in daily life. [1][2]

It is now well known the response of closed-loop system is much faster than the open-loop. Thus more mathematical methods were developed to solve the closed-loop control, however they are not straightforward. [3]

A system's performance will be greatly influenced by the position of the poles, especially by a pair of dominant poles. Since conventional mathematical techniques are too complex to effectively analyze the system, new graphical methods have been proposed to analyze the performance of control system. [4]

In [5], Kinnen proposes a graphical control method to control multivariable control systems, a significant breakthrough in the control area. Compared with the traditional method (utilizing matrix formulation), the calculation is greatly reduced. In [6] O'Brien attempts to design a discrete control system, simply based on the Root Locus method. In [7], Koenig worked on analyzing a system's frequency response and then determining the stability of the control system using a Bode diagram. In [8] Hla Myo Tun used a Matlab Bode plot to analyze the performance of a lead-lag compensator. Similarly, another frequency tool, the Nyquist diagram, is used to analyze the stability of control system by Jones in [9].

This paper focusses on an improved process to design the system in a simple way and analyze which graphical technique should be used in different cases. 


\section{The Concept of Control}

\subsection{Important Parameter for Control System}

To design a robust control system, it is necessary to know several important parameters. These parameters can reflect the system's performance.

Settling time: settling time is defined as the time for the response to reach and stay within $2 \%$ of its final value. [10]

$$
T_{s}=\frac{4}{\zeta \omega_{n}}
$$

Percentage of Overshoot: percentage of overshoot is defined by the amount that the waveform overshoots the steady-state or final value at the peak time, expressed as a percentage of the steadystate value. [11]

$$
\% O S=e^{\frac{-\zeta \pi}{\sqrt{1-\zeta^{2}}}} * 100
$$

Steady-state error: Steady-state error is defined as the difference between the input and the output when the system reaches a steady state. Assuming the test input is a step function of a time: [10]

$$
E_{s s}=1-\frac{K * Z_{1} * Z_{2} \cdots}{p_{1} * p_{2} \cdots}
$$

Peak time: Peak time is the time required to reach the first, or maximum, peak. [11]

$$
T_{p}=\frac{\pi}{\omega_{n} \sqrt{1-\zeta^{2}}} \quad T_{p}=\frac{\pi}{\omega_{n} \sqrt{1-\zeta^{2}}} \quad T_{p}=\frac{\pi}{\omega_{n} \sqrt{1-\zeta^{2}}}
$$

\subsection{Root Locus}

Root Locus is graphical method that was developed by Walter R.Evans to analyse and design control systems. The Root Locus is always used to plot the poles of the closed loop transfer function whose gain is variable.

To draw the Root Locus Diagram of negative feedback system with less calculation, there are five rules to follow.

Rule 1: The number of closed-loop poles equals the number of the branch of the Root Locus. The closed-loop pole moves if the gain is changed. So each pole will have its own path due to the change of the gain.

Rule 2: The diagram of the Root Locus is symmetrical about the real axis.

Because normal systems can only have the real number of coefficients in the transfer function, the closed-loop poles must exist in conjugate pairs. That causes the symmetry of the Root Locus.

Rule 3: The Root Locus begins at the finite and infinite poles of $\mathrm{G}(\mathrm{s}) \mathrm{H}(\mathrm{s})$ and ends at the finite and infinite zeros of $\mathrm{G}(\mathrm{s}) \mathrm{H}(\mathrm{s})$.

Rule 4: For $\mathrm{K}>0$, the Root Locus has branches at the left of an odd number of real-axis, finite open-loop poles and/or finite open-loop zero.

Rule 5: The Root Locus approaches straight lines when the locus approaches infinity. The line is given by the real axis intercept, $\sigma_{\mathrm{A}}$, and angle is $\theta_{\mathrm{A}}$ as follows: 


$$
\begin{gathered}
\sigma_{A}=\frac{\sum \text { Finite poles }-\sum}{\text { Number of Finite Poles }-N} \sigma_{A}=\frac{\sum \text { Finite poles }-\sum \text { Finite Zeros }}{\text { Number of Finite Poles }- \text { Number of Finite Zeras }} \\
\qquad \vartheta_{A}=\frac{(2 k+1) \pi}{\text { Number of Finite Poles }- \text { Number of Finite Zeros }} \quad k=0 ; \pm 1 ; \pm 2
\end{gathered}
$$

\subsection{Frequency Design Tools}

Nyquist Diagram and Bode Diagram are two popular frequency design tools used in the control system. The most common use for the Nyquist plot is to analyse the stability of the control system whereas the Bode diagram is used to design the control system.

Before plotting the diagrams using frequency design tools, phasors must be properly understood. Phasors are defined as a vector which is used to represent a sinusoid. A sinusoid consists of the magnitude and its phase angle, thus the phasors also has its own magnitude and phase angle.

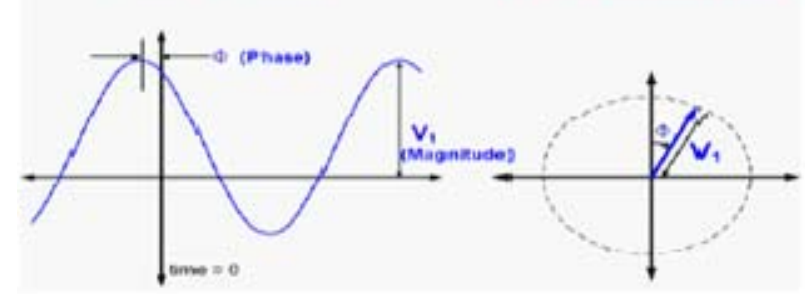

Figure 1 Sinusoidal waveform and its phasor representation [12]

Frequency response diagrams can be plotted in separate diagrams or in one diagram. The separate plot contains two diagrams: magnitude vs. frequency and phase vs. frequency. When plotting the frequency response on one graph, the length of the phasors is the magnitude and angle is phase.

\subsubsection{Bode Diagram}

The Bode diagram can be defined as two separate plots about log-magnitude and phase curve as functions of $\log \omega$. To simplify calculation, the Bode diagrams always be plotted as a sequence of straight lines.

Suppose the transfer function of $\mathrm{G}(\mathrm{s})$ :

$$
G(s)=\frac{K\left(s+z_{1}\right)\left(s+z_{2}\right) \ldots\left(s+z_{m}\right)}{s^{t}\left(s+p_{1}\right) \ldots\left(s+p_{n}\right)}
$$

The magnitude frequency response:

$$
|G(s)|=\lim _{s \rightarrow j \omega} \frac{|K|\left|\left(s+z_{1}\right)\right|\left(s+z_{2}\right)|\ldots|\left(s+z_{m}\right) \mid}{\left|s^{t}\right|\left|\left(s+p_{1}\right)\right| \ldots||\left(s+p_{n}\right) \mid}
$$

The phase frequency response will equal the phase curve of zeros minus the phase curve of poles.

\subsubsection{Nyquist Diagram}

In order to correctly and easily plot the Nyquist Diagram, two important concepts must be introduced:

1: the poles of $1+\mathrm{G}(\mathrm{s}) \mathrm{H}(\mathrm{s})$ and the poles of $\mathrm{G}(\mathrm{s}) \mathrm{H}(\mathrm{s})$ are the same.

2: the poles of $\mathrm{T}(\mathrm{s})$ are equal to the zeros of $1+\mathrm{G}(\mathrm{s}) \mathrm{H}(\mathrm{s})$.

After introducing two basic concepts, right-hand contour would be used to help to complete Nyquist Diagram. 

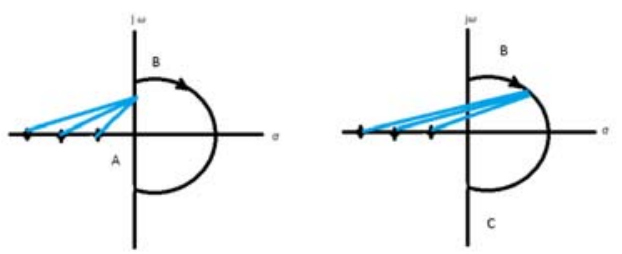

Figure 2 Nyquist diagram for low frequency (left) and high frequency (right)

Plotting procedure:

1: Determine the transfer function of $G(j \omega)$.

2: Multiply the numerator and denominator by the complex conjugate of the denominator.

3: Set $\omega=0$ and get the starting point, then find the point that pass imaginary axis and real axis, last find out the point that $\omega$ tends to be infinite. (From path $\mathrm{A}$ to $\mathrm{B}$ )

4: Plot the diagram in high frequency part (path B to C, in Fig 4).

Note: sometimes the shape of right-hand contour may be changed because the open loop-pole may locate on the imaginary axis. Detour can be used to solve this problem. There are two kinds of detour: detour right and detour left(in Fig. 3).
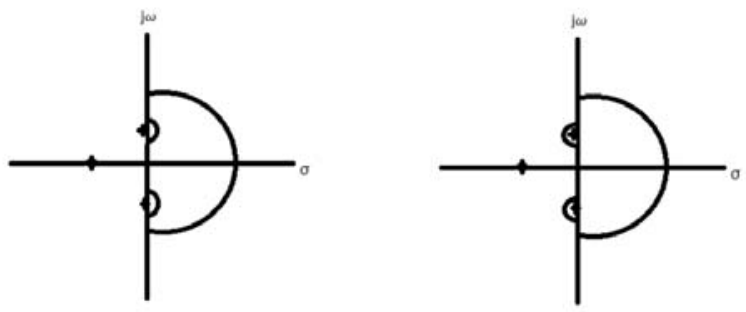

Figure 3 Detour right and detour left

Generally speaking, the Nyquist diagram is not used to design the control system, however it is widely used to analyse the stability of the system.

\subsection{Stability Study}

Normally, the Nyquist diagram can be used to find the critical value of gain to keep the system stable. Before using the Nyquist criterion to determine the stability of the control system, mapping rules will be introduced.

Rule 1: The direction for mapping will not change if it just has zeros and poles not encircled by the contour. (shown in Fig. 4)
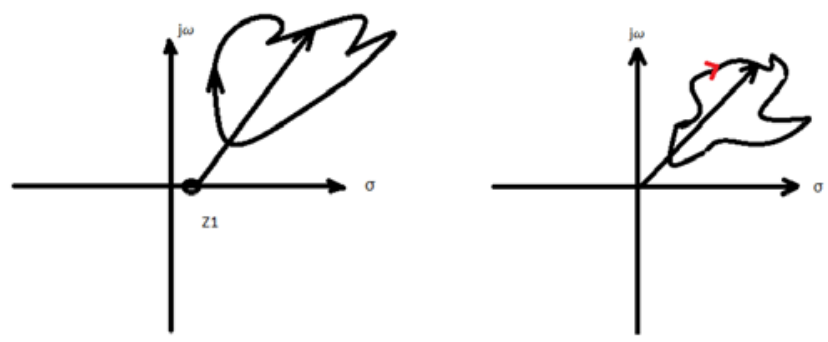

Figure 4 Mapping counters for zeros outside of the circle

Rule 2: The direction of mapping contours will not change and the mapping encircles the origin and has zeros encircled by the contour.

Rule 3: The direction of mapping contours will change if both mapping encircles the origin and the poles are encircled by the contour.

Rule 4: Poles and zeros can cancel the effect of each other.

The Definition for Nyquist stability: If for a contour A, when mapped through G(s) H(s), then the number of closed loop poles $\mathrm{Z}$ in right half plane(these poles are unstable) is given by (9).

$$
\mathrm{Z}=\mathrm{P}-\mathrm{N}
$$

By calculation the number of $Z$, the stability of the system can be analysed. 
Thus, the stability by the Nyquist Diagram can be summarised by:

Step 1: Sketch the Nyquist Diagram of $G(j \omega) H(j \omega)$, by set the value K as 1 .

Step 2: Calculate the value of $Z$, but in this case, $N$ equals to the number of counter clockwise rotation around $-1 / \mathrm{K}$.

Step 3: Change the value of $K$ and find the critical value of $K$ that keep the system stable.

Sometimes to analyse the stability of the system, Bode diagram can also be used. Compared with the Root Locus and Nyquist diagrams, Bode Diagrams are easy to implement. Calculation is much reduced by the Bode diagram; however the result for this method is not as accurate as Root Locus and Nyquist.

\subsection{Popular Compensator}

An ideal compensator uses pure integration or pure differentiation to improve the steady-state error or transient response of a system. Some of ideal compensators (PI and PID controller) can reduce the steady-state error to zero, but all ideal compensators need active circuit and extra power.

Compared with the ideal compensator, the non-ideal compensators are more energy saving and easy to implement. Thus the cost of non-ideal compensator is cheap; however the design for nonideal compensator is difficult. The performance of non-ideal compensators is worse than the ideal compensator. There are three kinds of non-ideal compensators: Lead, Lag and Lead-Lag compensator. all of them are implemented by carefully choosing the compensators' pole and zero.

\section{Method to Design Controllers}

Generally speaking, in modern control theory, there are two popular methods to compensate the system: cascade compensation and feedback compensation. Compared with the cascade compensation, feedback compensation is much more difficult to design. Both of them will change the closed-loop poles and zeros of the system, thus the system will have new performance.

\subsection{Design via Root Locus}

Root Locus is a graphical method to design the system, mostly used to design the system with variable gain. Due to the nonlinear relationship between gain and pole location, it is necessary to define the sensitivity for the control system. High sensitivity system is defined as: the small gain change leads to large change for the pole position. Otherwise, the system is defined as the low sensitivity system. In a real control system, low sensitivity system is required.

\subsubsection{PID Controller}

The design procedure for PID controller:

Step 1: Plot the Root Locus and evaluate the uncompensated system. Then determine the improvement required in the transient response.

Step 2: Deal with the transient response with a PD controller. Simulate the system with the PD controller and check the effect of the PD controller.

Step 3: Redesign the system if the requirement cannot be met.

Step 4: Design the PI controller using the technique stated before to meet the requirement of steady-state error. Determine the gains of system.

Step 5: Use simulation software, such as simulink, to simulate the whole system to make sure that all the requirements are met.

Step 6: Redesign if the system cannot meet the requirement.

\subsubsection{Lead-Lag Compensator}

The basic ideal for the Lead-Lag compensator is to use a Lead compensator to improve the transient response and a Lag compensator to improve steady-state error. This system will introduce two poles and two zeros, and the calculation is difficult compared with the PID controllers.

The design procedure for Lead-Lag compensator: 
Step 1: Plot the Root Locus of uncompensated system and determine the improvement required in the transient response.

Step 2: Find a Lead compensator to improve transient response. The design should cover the position of pole and zero.

Step 3: Simulate the system with the Lead controller and check the effect of the Lead Controller. Redesign the system if the requirement cannot be met.

Step 4: Calculate the adjustment required in the steady-state error. Design a Lag controller using the technique stated before to meet the requirement of steady- state error.

Step 5: Use simulation software, such as Simulink the whole system to make sure that all the requirements are met.

Step 6: Redesign if the system cannot meet the requirement.

The Root Locus is easy to implement, however this method has several limitations. The significant disadvantage of this method is that only the points on the Root Locus, rather than s-plan, may be designed. The possible solutions may therefore be greatly limited.

\subsection{Design via Frequency Tools}

There are two frequency tools: Nyquist diagram and Bode diagram. However, Nyquist diagram is very complicated when it is used to design the system. Instead, Inverse Nyquist is usually used to design the control system. In this section, the system will be designed by using a Bode diagram and an Inverse Nyquist diagram. Bode diagram design will be implemented by Matlab code and Inverse Nyquist design will be implement by a hand-drawn diagram.

\subsubsection{Bode Diagram}

Compared with the Root Locus, there is a slight change in the design procedure. The design procedure for Root Locus diagram is normally designing the transient response first and then designing the steady-state error. The Bode design inverses the design procedure, designing the steady-state error first, followed by the transient response. The system is always designed by a Lead-Lag compensator.

Lead-Lag compensator design via Bode diagram:

Step 1: Calculate the closed-loop bandwidth to meet the transient response by using second order approximation.

Step 2: Set the value of gain, K, to meet the requirement of steady-state error.

Step 3: Plot the log-magnitude and phase diagrams for the gain $(\mathrm{K})$ calculated before.

Step 4: In order to meet damping ratio or percentage of overshoot, calculate phase margin by using second order approximation.

Step 5: Choose a new phase margin frequency which is close to $\omega \mathrm{BW}$.

Step 6: Calculate the additional lead phase contribution required and add a small contribution to compensate the Lag compensator.

Step 7: Design the lag compensator first, followed by the lead compensator.

Step 8: Check the bandwidth to clarify all the requirements have been met. Redesign the system if the requirement cannot be met.

The Bode diagram is very easy to implement compared with other methods, however due to the approximation for the shape of the Bode diagram, this method is not precise compared with other methods.

\section{Experimental Study and Result Analysis}

When the plotting tools such as Matlab or angle calculation programs are not available, or the system does not require very precise design it is possible to use hand-drawn graphs to design the system. The first group of experiments will be set to compare the hand-drawn design method. 


\subsection{Design via Hand-drawn Diagram}

In this section, Root Locus, Bode Diagram and Nyquist diagram are used to analyse the performance of system. Then the diagram is used to design the required system t. Finally, the feasibility of each design work is analysed.

Assuming the open-loop transfer function of the control system that need to design is: $\frac{K}{s(s+1)(s+4)}$ and the system has unity feedback, then the design requirements for the system are:

1) Design a PD controller to reduce the settling time to be threefold of the original system. (Overshoot is $11 \%$ for original system)

2) Design a Lag compensator to reduce the steady-state error to be tenfold of the original system. (Overshoot is $9.5 \%$ for original system)

3) Using Nyquist Diagram to determine the critical value of $K$ to make the system stable.

\subsubsection{Design PD Controller}

First, use the Hand-drawn Root-Locus to design a PD controller. Fig.5. shows the design procedure.

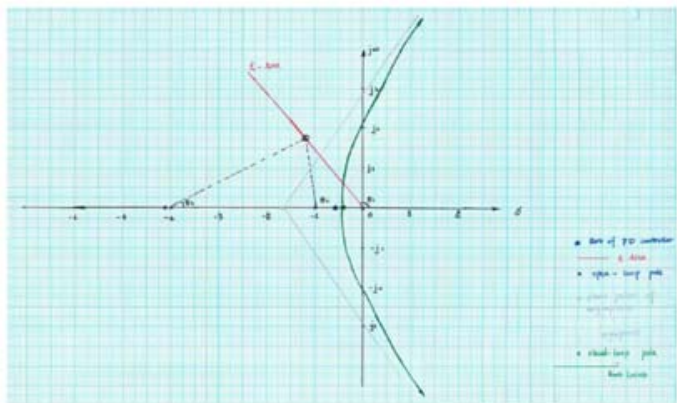

Figure 5 Root Locus to design and analyse the control system

After careful design, the transfer function for PD controller is:

$$
G_{P D}(s)=s+0.597
$$

This method is easy to implement and the calculation is very simple: the only equipment required is a protractor.

From the design perspective, Fig. 7 clearly shows the dominant pole in the graph, however, the third order pole or higher order poles are not clearly shown in the graph. This does not matter particularly, because the performance of the system mainly is depending on the dominant poles.

There are three limitations of this method:

The first limitation comes from the precise of measurement. Using the protractor to measure the phase angle will lead to the error and this error cannot be ignored, especially for the low order system.

Secondly, even though the dominant poles may be obtained in the graphic directly, the operating gain can not be easily determined for the system, making it difficult to simulate the system in software.

Finally, engineers can only design the system on the Root Locus or the $\zeta$ line, meaning alternative solutions may be missed.

\subsubsection{Design a Lag Compensator}

In this section, Hand-drawn Bode Diagram is to be used to design a Lag compensator to reduce the steady state error.

After calculation, the transfer function for Lag compensator is:

$$
G_{\text {Lag }}(s)=\frac{0.875(s+0.024)}{(s+0.021)}
$$

Finally use software such as MATLAB's simulink to simulate the system. The result is shown in Fig. 6, demonstrating it meets the requirement. 


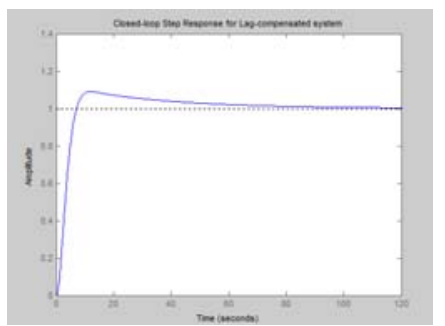

Figure 6 The Closed-loop Step Response for Lag-compensated system

This design is somewhat complex compared with the Root Locus, because the design is based on two graphs rather than one. The calculation for this diagram is extremely easy compared with other method. There is no particular equipment required in the design procedure.

The advantage of plotting is that the stability of the system can be read directly from the diagram and the operation gain can be acquired by simple calculation. This diagram also can be acquired by simply measuring the output of the system. In this approach, knowledge of the open-loop transfer function is not required. This characteristic makes Bode diagrams extremely useful when the transfer function of the system is unavailable.

Due to the great error for simple pole or zero design, this method is always used to design the Lag compensator, Lead compensator or Lead-Lag compensator rather than the PI, PD and PID controllers.

\subsubsection{Find the Critical value of Gain to Make System Stable}

The Nyquist Diagram can be used to analyse the stability of a control system. Follow the steps mentioned in part 3 , section $\mathrm{D}$, the critical value of gain that results in a stable system is 20 . The diagram is shown in Fig. 7.

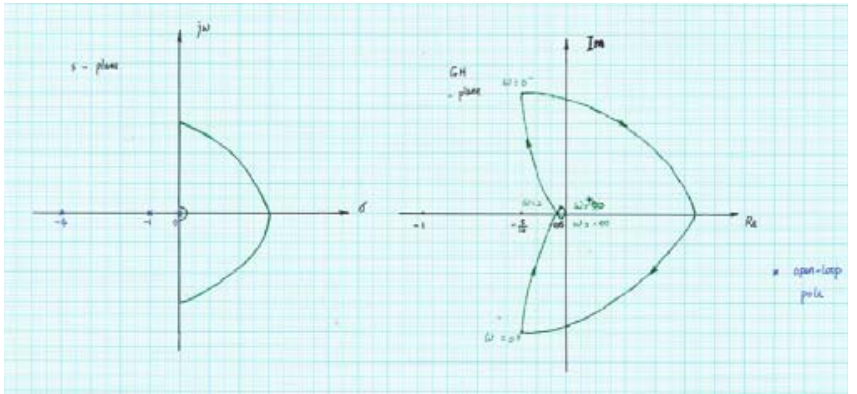

Figure 7 Nyquist diagram for the original open loop system (Suppose the gain of the system is 1)

Although the Nyquist Diagram is very useful when analysing the stability of a system, this method does not give a lot of additional information. So this method is not used to design the control system. However, the diagram is clear compared with Bode diagram and the result of stability analysis is precise.

\subsection{Design via Matlab Coding}

Because more precise graphics can be drawn by the Matlab program, more complex compensators or controllers can be designed. In this part, for the same system as part A, a lead-lag compensator would be designed by Bode diagram to reduce overshoot and settling time. The usage of the compensator is to generate $13.25 \%$ overshoot, 2 seconds peak time.

The Lag compensator designed by the Matlab code is:

$$
G_{\text {Lag }}=\frac{0.094027(s+0.1828)}{(s+0.1719)}
$$

The Lead compensator designed by the Matlab code is:

$$
G_{\text {Lead }}=\frac{10.635(s+0.5605)}{(s+5.962)}
$$

The equivalent transfer function for the system is: 


$$
G e=\frac{48(s+0.1828)(s+0.5606)}{s(s+4)(s+5.962)(s+1)(s+0.01719)}
$$

The Simulink result is shown in Fig. 8.

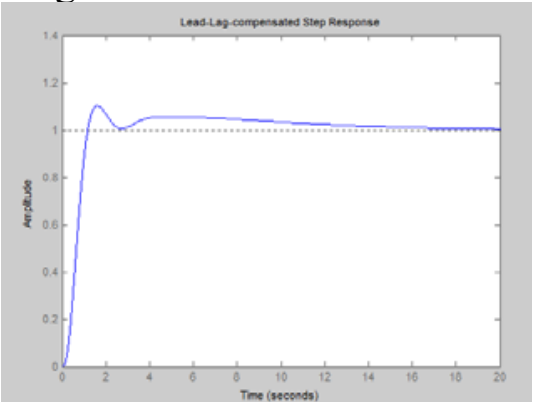

Figure 8 Lead-Lag-compensated Step Response

This system is identical to the previous system. However, compared with the previous system, this design performs much better and the result is more accurate. The settling time for this system is around one-sixth of the previous system and the peak time is only $2 \mathrm{~s}$. In other words, the transient response is better. In this program, the compensators both improve the steady-state error and the transient response.

Because this method does not involve plotting, extra useful information cannot be acquired, so the function of this method is somewhat limited. Compared with the hand-drawn method, this approach can only be used to analyse a linear system, but is not suitable to design non-linear systems and discrete time systems. The performance of the system will be strictly influenced by the coding, thus a good programming will produce a more accurate result. Designing a good program is the only difficulty of this method.

\section{Conclusion and Future Work}

Graphical design is a more simple way to design the control system. Compared with the mathematical design or traditional design methods, the calculation has been greatly minimized. At the same time, graphical design methods provide more alternative solutions when designing one control system; so the best result may be chosen.

The first group of experiments show the design procedure without any help from calculation tools and software, are very easy to implement and the result can be satisfied as anticipated. The second group of experiments has the same conditions as the first group; but are used to compare Matlab design vs. hand-drawn design. From the simulation result, it is obviously true that Matlab performs much better. The last group of experiments is designed to analyse the difference between feedback design and cascade design. Obviously, feedback design can offer more precise design compared with the cascade.

In the future, in order to prove the excellence of graphical control, a program should be designed to compare the result of graphical design and mathematical design. Thus the importance of graphical design can be highlighted.

\section{References}

[1] Modern Control Engineering, Katsuhiko Ogata, Fourth Edition, Prentice-Hall, copyright 2002, page 1-8

[2] Modern Control Engineering, Katsuhiko Ogata, Fifth Edition, Prentice-Hall, copyright 2010, page11-20

[3] Control system technology, C.J.Chesmond, Second Edition, Q Search, copyright 1982, page2-11

[4] Control Systems Engineering, Norman S.Nise, Fifth edition, John Wiley, copyright 2008, page $371-372$ 
[5] Linear multivariable control system design with root loci, Kinnen, E, American Institute of Electrical Engineers, Part II, IEEE

[6] A unified procedure for discrete-time root locus and Bode design, O'Brien, R.T., American Control Conference, 2005. Proceedings of the 2005, IEEE

[7] The bode diagram: An approach to regulating-system stability fundamentals, Koenig, L. A., Electrical Engineering, IEEE

[8] Design analysis of phase lead compensation for typical laser guided missile control system using MATLAB Bode plots, Hla Myo Tun, Control, Automation, Robotics and Vision, 2008. ICARCV 2008. 10th International Conference on, IEEE

[9] Stability of feedback systems using dual Nyquist diagram, Jones, Paul, Circuit Theory, IRE Transactions, IEEE

[10] Control Systems Engineering, Norman S.Nise, Fifth edition, John Wiley, copyright 2008, page169

[11] Steady State Error In Control Systems, Bucknel University http://www.facstaff.bucknell.edu/mastascu/econtrolhtml/Design/Perf1SSE.html, accessed $06 / 03 / 2013$

[12] CERTS. http://www.phasor-rtdms.com/phaserconcepts/phasor_adv_faq.html , accessed 04/05/2013 